\section{Transfusion Medicine and Hemotherapy}

\title{
3. Marburger Transfusionsgespräche, 13. und 14. Februar 2004
}

Am 13. und 14. Februar 2004 fanden in Marburg die «3. Marburger Transfusionsgespräche» statt. Die Veranstaltung beruht auf einer langjährigen transfusionsmedizinischen Tradition, die erstmals bereits 1966 von H. Zöckler als «Bremer Transfusionsgespräche» gegründet wurde und dort bis 1983 insgesamt zehnmal stattfand. Nach mehrjähriger Pause wurde ab 1989 der Gedanke eines regelmäßigen Erfahrungsaustauschs in der Transfusionsmedizin in Form der «Transfusionsmedizinischen Gespräche Hannover» als jährliche Veranstaltung erneut aufgegriffen und bis 1998 dort fortgeführt. Von 1999-2001 wurden die Gespräche in Kassel veranstaltet, seit 2002 finden sie unter der Organisation von V. Kretschmer in Marburg statt.

Das Ziel dieser Veranstaltung besteht im gegenseitigen Erfahrungsaustausch von Transfusionsmedizinern und Klinikern, die insbesondere als Transfusionsverantwortliche und Transfusionsbeauftragte gemäß Transfusionsgesetz in klinischen Einrichtungen tätig sind. Die Veranstaltung war mit 170 Teilnehmern, davon zirka 140 Kliniker, gut besucht. Zunächst wurde durch einen Vertreter des Bundesministerium für Gesundheit (F. von Auer) über neue rechtliche Vorgaben informiert und insbesondere die anstehende Novellierung des Transfusionsgesetzes ausgiebig diskutiert. Anschließend berichteten Kollegen aus Wien (P. Perger), Marburg (T. Zeiler) und Jena (D. Barz) über Transfusionszwischenfälle und die Entwicklung des transfusionsmedizinischen Qualitätsmanagements. In diesem Zusammenhang wurde speziell auch die Bedeutung des AB0-Bedside-Tests (T. Hoffmann, Düsseldorf) diskutiert. Mit der Einführung der DRGs ergeben sich zusätzliche organisatorische und ökonomische Probleme, die vom ersten Vorsitzenden der DGTI, N. Müller (Essen), erläutert wurden. Mit besonderem Interesse wurde die Transfusion von Neugeborenen diskutiert, über die R.F. Maier (Marburg) referierte. Wie schwierig die rechtlichen Vorgaben im Alltag umgesetzt werden können, zeigten die Erfahrungsberichte von Qualitätsbe- auftragten (V. Heymann, Berlin; T. Rogge, Berlin; D. Mix, Darmstadt). Die Sitzung über autologe Hämotherapie dokumentierte, welch großes Interesse unverändert von Seiten der Kliniker diesbezüglich besteht. D.V. Dietrich (Eggenfelden) gab eine Einschätzung hinsichtlich der Bedeutung der präoperativen Eigenblutspende, die zeigte, dass diese nach wie vor eine wichtige Maßnahme zur Vermeidung von Fremdbluttransfusionen darstellt. Dagegen sah E. Hansen (Regensburg) keinen Sinn mehr in der Verwendung von ungewaschenem Wundblut.

In einer weiteren Sitzung wurden Möglichkeiten aufgezeigt, wie die notwendigen Maßnahmen zur Qualitätssicherung in der Klinik umgesetzt werden können. T. Hoffmann (Düsseldorf) berichtete über die Tätigkeit von Transfusionskommission und transfusionsmedizinischem Arbeitskreis in seiner Klinik. G. Rump (Ludwigshafen) informierte über eine Umfrage, die bezüglich der Hospitation von Tranfusionsverantwortlichen in transfusionsmedizinischen Einrichtungen von ihm durchgeführt wurde und ein Defizit insbesondere bezüglich hämostaseologischer Fortbildung erkennen ließ. G. Dinges (Marburg) und V. Kretschmer (Marburg) referierten über Fragen der Depotführung unter Berücksichtigung des Blutkonservenbedarfsplans für operative Standardeingriffe. Die Veranstaltung wurde durch ein Referat zur Antikoagulation in der Klinik (B. Pötzsch, Bonn), die der hämostaseologischen Fortbildung diente, abgerundet.

Insgesamt wurde engagiert, offen und auch kontrovers diskutiert, wobei immer wieder deutlich wurde, wie schwierig theoretische Forderungen unter Routinebedingungen umzusetzen sind; dies kann am ehesten durch den intensiven Erfahrungsaustausch zwischen Klinikern und Transfusionsmedizinern realisiert werden. Die Veranstaltung wurde daher von den Teilnehmern sehr positiv beurteilt, so dass auch die nächste Veranstaltung, die «4. Marburger Transfusionsgespräche» am 11. und 12. Februar 2005, großes Interesse erwarten lassen. 